\title{
Planejamento experimental no estudo eletroquímico de camadas de óxido de nióbio em liga de alumínio 3003
}

\section{(Experimental design for electrochemistry study of niobium oxide layers in 3003 aluminum alloy)}

\author{
M. Tussolini ${ }^{1,2}$, T. Ichikawa ${ }^{1}$, A. L. Gallina ${ }^{1,3}$, A. Viomar', A. C. Antunes ${ }^{4}$, M. T. Cunha ${ }^{1}$, \\ E. P. Banczek ${ }^{1}$, P. R. P. Rodrigues ${ }^{1}$ \\ ${ }^{I}$ Departamento de Química, UNICENTRO, R. Simeão C. V. Sá 03, Guarapuava, PR, 85040-080 \\ ${ }^{2}$ Instituto Federal do Mato Grosso - IFMT, Campus Barra do Garças, BR-158 s/n, M T 78.600-00 \\ ${ }^{3}$ Universidade Federal da Fronteira Sul, Campus Realeza, R. Edmundo Gaievski 1000, Realeza, PR \\ ${ }^{4}$ Departamento de Química, UEPG, Campus Uvaranas, PR 84030-900 \\ martha.tussolini@bag.ifmt.edu.br
}

\begin{abstract}
Resumo
A cromagem é uma opção que a indústria de metal mecânica tem encontrado para aumentar a resistência à corrosão de peças metálicas, porém esse revestimento apresenta a desvantagem de ser tóxico ao ser humano e ao meio ambiente. Para se alcançar um novo tratamento de superfície, pode-se aplicar planejamento experimental, a fim de otimizar os experimentos, principalmente quando há diversas variáveis para se chegar ao processo ou produto final. O objetivo principal deste trabalho é utilizar planejamento experimental para se obter um revestimento cerâmico à base de óxido de nióbio para ligas de alumínio 3003 e compará-lo ao revestimento de $\mathrm{Cr}$ (III), no mesmo substrato metálico. As técnicas empregadas foram polarização potenciodinâmica anódica, espectroscopia de impedância eletroquímica, microscopia eletrônica de varredura e mapeamento por espectroscopia de energia dispersiva. Utilizou-se um planejamento experimental $2^{3}$, no qual as variáveis foram velocidade de imersão, tempo de repouso após imersão e emersão na solução sol-gel à base de óxido de nióbio (razões molares n/n ácido cítrico/etilenoglicol 1:4, ácido e o complexo amoniacal de nióbio 10:1). Registrou-se uma tendência nas superfícies de respostas estudadas, sugerindo que em 5 min de imersão e emersão e na velocidade de $18 \mathrm{~cm} / \mathrm{min}$, o filme gerado apresenta uma maior resistência à corrosão. Os resultados de impedância eletroquímica e polarização potenciodinâmica anódica mostraram que o filme de óxido de nióbio apresenta maior resistência à corrosão que o revestimento utilizado industrialmente. Sendo assim, esse novo tratamento de superfície torna-se uma opção de substituição do revestimento a base de Cr (III).
\end{abstract}

Palavras chave: cerâmica, revestimento, tratamento de superfície.

\begin{abstract}
The plating is an option that the metalworking industry has been found to increase the corrosion resistance of metal parts, but this coating is toxic to humans and to the environment. To achieve a new surface treatment the experimental design may be applied to optimize the experiments, especially when there are many variables to obtain at the final product or process. The main objective of this paper is to use experimental design to obtain a ceramic coating based on niobium oxide over 3003 aluminum alloys and to compare it to the $\mathrm{Cr}$ (III) coating, in the same metallic substrate. The techniques employed were anodic potentiodynamic polarization, electrochemical impedance spectroscopy, scanning electron microscopy and mapping by energy dispersive spectroscopy. An experimental design $2^{3}$ was used, where the variables were sol-gel sink rate, settling time after immersion and emersion in the base solution of niobium oxide (molar ratios $n / n$ citric acid/ethylene glycol 1:4 acid and niobium ammonium complex 10:1). A tendency on the responses of the surfaces was detected, suggesting that at 5 min of immersion and emersion time and speed of $18 \mathrm{~cm} / \mathrm{min}$, the film achieved a higher corrosion resistance. The results of potentiodynamic polarization and electrochemical impedance spectroscopy showed that the anodic film of niobium oxide is more resistant than the coating used industrially, so that new surface treatment becomes a replacement option for Cr (III) coating.
\end{abstract}

Keywords: ceramic, coating, surface treatment.

\section{INTRODUÇÃO}

A resistência à corrosão é uma das importantes propriedades dos metais, pois quando expostos a meios agressivos tendem a sofrer processos corrosivos $[1,2]$.
Existem diversos tratamentos de superfície que tendem a retardar os processos de oxidação $[3,4]$, podendo-se destacar a cromatização, onde a resistência à corrosão é bastante elevada. O elemento cromo apresenta como desvantagem ser prejudicial à saúde e ao meio ambiente, da mesma forma que 
os revestimentos a base de níquel. Atualmente, buscam-se soluções ao uso destes revestimentos que inibam à corrosão e que sejam menos poluentes $[5,7]$. Registra-se desta forma a necessidade de desenvolvimento de novos tratamentos de superfícies eficientes e com matéria prima de fácil acesso. Um dos elementos que tem se mostrado bastante interessante para novas pesquisas é o nióbio. O Brasil detém a maior reserva mundial de nióbio, tendo aproximadamente $98 \%$ da produção mundial [4].

O nióbio tem sido empregado em diferentes finalidades, como materiais para absorção de luz UV [9], para troca iônica $[10,11]$, catalisador [12], entre outros. Há inúmeras rotas que podem ser utilizadas para o depósito de óxido de nióbio, sendo as mais empregadas aspersão térmica [13], fase vapor [14] e sol gel [15-18]. Para essa última via, podemse optar pelo método Pechini [19-22] Esse método consiste na utilização de um ácido orgânico hidrocarboxílico para formação de um quelato com um cátion. Ao quelato adicionase um poliálcool e, por via de reação de condensação, se obtém um éster. Em uma segunda etapa, é feito um tratamento térmico para eliminar a fase orgânica, obtendo-se assim o produto desejado. Este método tem sido utilizado para obter diversas cerâmicas [19, 23-27]. As grandes vantagens desse método são proporcionar uma boa homogeneidade química dos multicomponentes em escala molecular [28], controle direto e preciso da estequiometria em sistemas complexos, e a presença de água para não prejudicar a obtenção do produto final [23].

As diferentes etapas realizadas até a obtenção da cerâmica influenciam em sua morfologia, conseqüentemente sua aplicação. Planejamentos experimentais para estudos de otimização do processo de obtenção de revestimentos tem sido amplamente empregados, iniciando em pesquisas laboratoriais e se desdobrando em desenvolvimentos industriais [29]. Com o delineamento de variáveis, podem-se verificar as propriedades de interesse que são afetadas pela modificação das condições de estudo. Esses delineamentos permitem obter os parâmetros ideais para a otimização dos filmes com as melhores características nas aplicações de interesses [30].

$\mathrm{O}$ presente trabalho tem como objetivo aperfeiçoar o depósito de óxido de nióbio via recobrimento por imersão (dip coating) em liga de alumínio AA 3003, avaliando eletroquimicamente a resistência à corrosão desse novo revestimento cerâmico e comparando-o com o tratamento de superfície de $\mathrm{Cr}$ (III).

\section{MATERIAIS E MÉTODOS}

\section{Preparo das amostras e deposição dos filmes}

Os substratos metálicos empregados foram placas no formato quadrado, com dimensões $2 \times 2 \mathrm{~cm}$, em liga de alumínio 3003. O processo de tratamento de superfície prévio nos corpos de prova foi o lixamento com lixas de $\mathrm{SiC}$ de 320, 400, 600 e 1200 mesh.

O preparo da resina foi realizado pela dissolução de ácido cítrico $\left(\mathrm{C}_{6} \mathrm{H}_{8} \mathrm{O}_{7}\right)$ em etilenoglicol $\left(\mathrm{C}_{2} \mathrm{H}_{4}(\mathrm{OH})_{2}\right)$ a $60{ }^{\circ} \mathrm{C}$ sob agitação magnética, com posterior adição do complexo amoniacal de nióbio. A solução resultante foi mantida sob agitação por $30 \mathrm{~min}$. A resina teve razões molares $(\mathrm{n} / \mathrm{n})$ ácido cítrico:etilenoglicol 1:4, e entre o ácido e o complexo amoniacal de nióbio 10:1. As cerâmicas de óxido de nióbio foram depositadas via imersão das amostras na resina. Os filmes foram submetidos a dois tratamentos térmicos, a 100 ${ }^{\circ} \mathrm{C}$ por $60 \mathrm{~min}$ e posteriormente a $450{ }^{\circ} \mathrm{C}$ por $30 \mathrm{~min}$.

Para efeito de comparação com o novo revestimento à base de óxido de nióbio, foi preparado um revestimento de $\mathrm{Cr}$ (III) comercial, depositado conforme recomendação do fabricante, sobre liga de alumínio 3003.

\section{Planejamento experimental}

Foi realizado um planejamento experimental $2^{3}$ com o programa Design-Expert ${ }^{\mathbb{}}$. As variáveis consideradas foram velocidade de imersão (velocidade) controlada por dip coating, tempo de repouso após imersão (imersão) e emersão (espera) da solução sol gel a base óxido de nióbio. Foram realizadas 15 deposições, repetidas no mínimo três vezes. Os resultados obtidos foram confrontados com os respectivos níveis de significância estabelecidos em 95\%. Com as respostas obtidas pelo planejamento experimental, foi feita modelagem teórica, visando obter o revestimento mais resistente à corrosão. O planejamento experimental está apresentado na Tabela I.

Tabela I - Matriz de planejamento experimental $2^{3}$ para verificar o efeito da velocidade de imersão, tempo de imersão e emersão no depósito do filme a base de óxido de nióbio.

[Table I - Matrix of $2^{3}$ experimental design to assess the effect of immersion speed, immersion and emersion time on the deposit of niobium oxide film.]

\begin{tabular}{cccc}
\hline Ensaio & $\begin{array}{c}\text { A } \\
\text { Imersão } \\
(\min )\end{array}$ & $\begin{array}{c}\text { B } \\
\text { Espera } \\
(\min )\end{array}$ & $\begin{array}{c}\text { C } \\
\text { Velocidade } \\
(\mathrm{cm} / \mathrm{min})\end{array}$ \\
\hline 1 & 5 & 10 & 18 \\
2 & 5 & 10 & 6 \\
3 & 30 & 10 & 18 \\
4 & 17,5 & 10 & 12 \\
5 & 30 & 0 & 18 \\
6 & 17,5 & 5 & 18 \\
7 & 17,5 & 5 & 12 \\
8 & 17,5 & 0 & 12 \\
9 & 5 & 0 & 6 \\
10 & 30 & 5 & 12 \\
11 & 5 & 0 & 18 \\
12 & 30 & 10 & 6 \\
13 & 17,5 & 5 & 6 \\
14 & 5 & 5 & 18 \\
15 & 30 & 0 & 12 \\
\hline
\end{tabular}




\section{Caracterização morfológica}

A morfologia da superfície da liga de alumínio AA 3003, sem e com revestimento, foi analisada por microscopia eletrônica de varredura (MEV), com aumento de 100 e 500x em um microscópio Tescan Vega3 Sem ${ }^{\circledR}$. O mapeamento da superfície foi determinado pela técnica de espectroscopia de energia dispersiva (EDS) Oxford ${ }^{\circledR}$.

\section{Caracterização eletroquímica}

Para a caracterização eletroquímica foi utilizada uma célula de três eletrodos, sendo o de trabalho (liga de alumínio AA 3003), o de referência (sulfato mercuroso saturado) e o contra eletrodo (platina de grande área), em $\mathrm{Na}_{2} \mathrm{SO}_{4}$ $0,5 \mathrm{~mol} / \mathrm{L}$ com adição de $800 \mathrm{mg} / \mathrm{L}$ de $\mathrm{NaCl}$, tamponado em $\mathrm{pH}=4,0$ com biftalato de potássio. Também foram realizados ensaios de potencial de circuito aberto em função do tempo $\left(\mathrm{E}_{\mathrm{CA}}\right)$ e curvas de polarização potenciodinâmica (PP) a partir do potencial de corrosão $\left(\mathrm{E}_{\text {corr }}\right)$ até 4 V/ESM, empregando velocidade de varredura de $5 \mathrm{mV} / \mathrm{s}$. As análises de espectroscopia de impedância eletroquímica (EIE) foram realizadas potenciostaticamente no $\mathrm{E}_{\text {corr }}$, com uma amplitude de perturbação $\pm 10 \mathrm{mV}$, na faixa de freqüência de $8 \mathrm{kHz}$ a $0,5 \mathrm{~Hz}, 10$ pontos por década para aquisição dos dados, em um analisador de resposta de freqüência Gamry EIS $300^{\circledR}$, acoplado a um potenciostato Gamry PCI $4 / 300^{\circledR}$.

\section{RESULTADOS E DISCUSSÃO}

Para avaliar o comportamento estacionário da liga de alumínio sem e com revestimento, foram realizados estudos do potencial de circuito aberto. Os valores dos $\mathrm{E}_{\text {corr }}$ para todos os ensaios do planejamento experimental estão apresentados na Tabela II.

As variáveis estudadas provocaram variações nos valores de $\mathrm{E}_{\text {corr }}$ Tais resultados são esperados, uma vez que alterando a deposição do revestimento, este apresentará diferença em sua morfologia, o que acarretará em mudanças em seu comportamento eletroquímico.

$\mathrm{Na}$ Tabela III estão apresentadas as respostas para o planejamento experimental. As respostas foram calculadas pela sobretensão (n) (Equação A) aplicada sobre as amostras revestidas até o rompimento do filme de óxido de nióbio em relação ao potencial registrado para o rompimento do filme (alumina) do substrato metálico. Determinouse o rompimento do filme no aumento indeterminado da densidade de corrente em cada ensaio de PP.

$$
\eta=\mathrm{E}_{\text {revestimento óxido de nióbio }}-\mathrm{E}_{\text {alumínio }}
$$

As respostas para todos os ensaios são positivas, mostrando que os rompimentos dos revestimentos de óxido de nióbio para todas as condições estudadas foram superiores ao do registrado para o substrato metálico, ou seja, $\eta$ positiva, evidenciando que os filmes formados são inibidores do processo de corrosão localizada (pites),
Tabela II - Valores dos $\mathrm{E}_{\text {corr }}$ das amostras de liga de alumínio 3003 revestidas com óxido de nióbio, a partir do planejamento experimental, em $\mathrm{Na}_{2} \mathrm{SO}_{4} 0,5 \mathrm{~mol} / \mathrm{L} \mathrm{em} \mathrm{pH} 4$ com $800 \mathrm{mg} / \mathrm{L}$ de $\mathrm{NaCl}$.

[Table II - Average values for $E_{\text {corr }}$ for 3003 aluminum alloy coated with oxide niobium, from experimental design, obtained in $\mathrm{Na}_{2} \mathrm{SO}_{4} 0.5 \mathrm{~mol} / \mathrm{L}$ in $\mathrm{pH} 4$ with $800 \mathrm{mg} / \mathrm{L}$ in $\mathrm{NaCl}$.]

\begin{tabular}{cc}
\hline Ensaio & $\begin{array}{c}\mathrm{E}_{\text {corr }}(\mathrm{mVvs} \\
\text { ESM })\end{array}$ \\
\hline 1 & $-947 \pm 3$ \\
2 & $-963 \pm 5$ \\
3 & $-913 \pm 4$ \\
4 & $-964 \pm 7$ \\
5 & $-1114 \pm 9$ \\
6 & $-971 \pm 4$ \\
7 & $-997 \pm 6$ \\
8 & $-1022 \pm 8$ \\
9 & $-1011 \pm 9$ \\
10 & $-1017 \pm 7$ \\
11 & $-1025 \pm 7$ \\
12 & $-913 \pm 5$ \\
13 & $-702 \pm 4$ \\
14 & $-935 \pm 5$ \\
15 & $-997 \pm 6$ \\
\hline
\end{tabular}

Tabela III - $\eta$ para os filmes obtidos nas condições do planejamento experimental $2^{3}$.

[Table III - $\eta$ for film obtained from experimental design $2^{3}$.]

\begin{tabular}{cc}
\hline Ensaio & $\begin{array}{c}\text { Resposta } \\
(\mathrm{V})\end{array}$ \\
\hline 1 & 2,1 \\
2 & 1,9 \\
3 & 2,8 \\
4 & 2,5 \\
5 & 1,4 \\
6 & 2,3 \\
7 & 2,8 \\
8 & 0,9 \\
9 & 1,1 \\
10 & 2,3 \\
11 & 1,8 \\
12 & 2,6 \\
13 & 2,7 \\
14 & 3,1 \\
15 & 2,3 \\
\hline
\end{tabular}

aumentando a resistência à corrosão do metal base.

A partir da análise das respostas do planejamento experimental $2^{3}$ apresentadas na Tabela IV, é possível avaliar 
os fatores principais, as interações, os respectivos efeitos e o p-valor.

Com $67 \%$ de confiança, o modelo apresenta como variável mais significativa o tempo de espera, porque essa variável interfere diretamente na espessura do filme, pois quanto maior for à espera, maior será o descarte da resina, resultando em um filme mais fino. Nota-se ainda na Tabela IV que alguns efeitos e interações apresentaram valores negativos, como o tempo de imersão e a velocidade, implicando que os aumentos dos valores dessas variáveis contribuem na diminuição do valor da $\eta$. De maneira distinta, os valores positivos do tempo de emersão mostram que maiores tempos de emersão colaboram para maiores valores nas $\eta$. $O$ valor de $\mathrm{R}^{2}$ foi 0,9856 , representando que $98,56 \%$ da variação total em torno da média é explicada pela regressão do modelo proposto. $\mathrm{O}$ erro padrão para todos os fatores e suas interações foi 0,16 e o modelo cúbico é o que melhor representou os valores experimentais. Com as respostas obtidas pelo planejamento experimental foi possível representar estatisticamente os resultados experimentais, apresentadas na Fig. 1.

Observa-se na Fig. 1 A-C que nos maiores tempos de imersão foram obtidos os menores valores de $\eta$. Isto ocorreu pois em maiores intervalos de tempo a resina à base de óxido de nióbio se decompôs. Outro fator que influenciou na resposta foi o tempo de emersão (espera), o que colaborou com a espessura do filme formado, onde a espera de $5 \mathrm{~min}$

Tabela IV - Valores dos efeitos principais e interações para o planejamento experimental $2^{3}$.

Table IV - [Values of the main effects and interactions for experimental design $2^{3}$.]

\begin{tabular}{ccc}
\hline $\begin{array}{c}\text { Fatores e } \\
\text { interações }\end{array}$ & Efeito & p-valor \\
\hline Modelo & 2,52 & 0,3334 \\
A- Imersão & $-0,44$ & 0,2828 \\
B- Espera & 0,77 & 0,1672 \\
C-velocidade & $-0,21$ & 0,4924 \\
AB & 0,078 & 0,5889 \\
AC & $-0,19$ & 0,3183 \\
BC & 0,077 & 0,5948 \\
$\mathrm{~A}^{2}$ & 0,26 & 0,3957 \\
$\mathrm{~B}^{2}$ & $-0,79$ & 0,1452 \\
$\mathrm{C}^{2}$ & 0,010 & 0,9651 \\
$\mathrm{ABC}^{2}$ & 0,20 & 0,3058 \\
$\mathrm{~A}^{2} \mathrm{~B}$ & $-0,42$ & 0,3214 \\
$\mathrm{~A}^{2} \mathrm{C}$ & 0,23 & 0,5072 \\
$\mathrm{AB}^{2}$ & 0,73 & 0,1972 \\
$\mathrm{AC}^{2}$ & - & - \\
$\mathrm{B}^{2} \mathrm{C}$ & - & - \\
$\mathrm{BC}^{2}$ & - & - \\
$\mathrm{A}^{3}$ & - & - \\
\hline
\end{tabular}

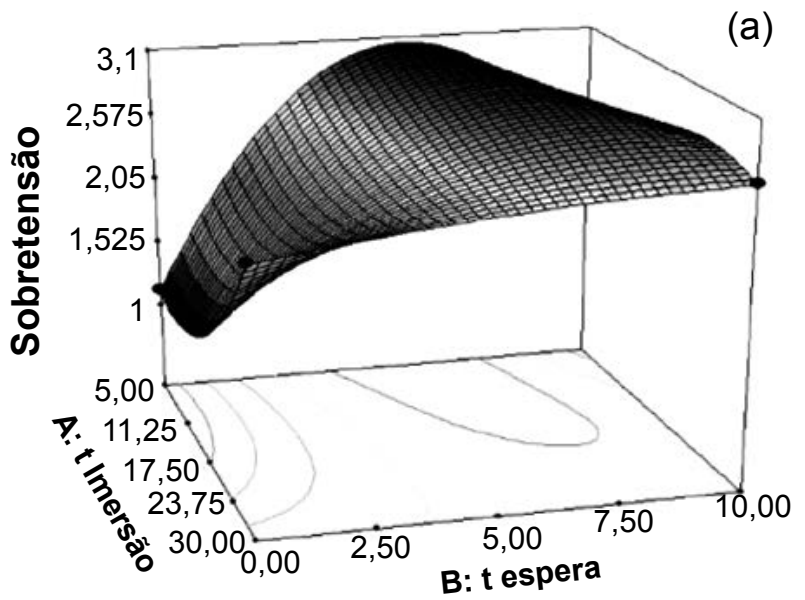

(b)
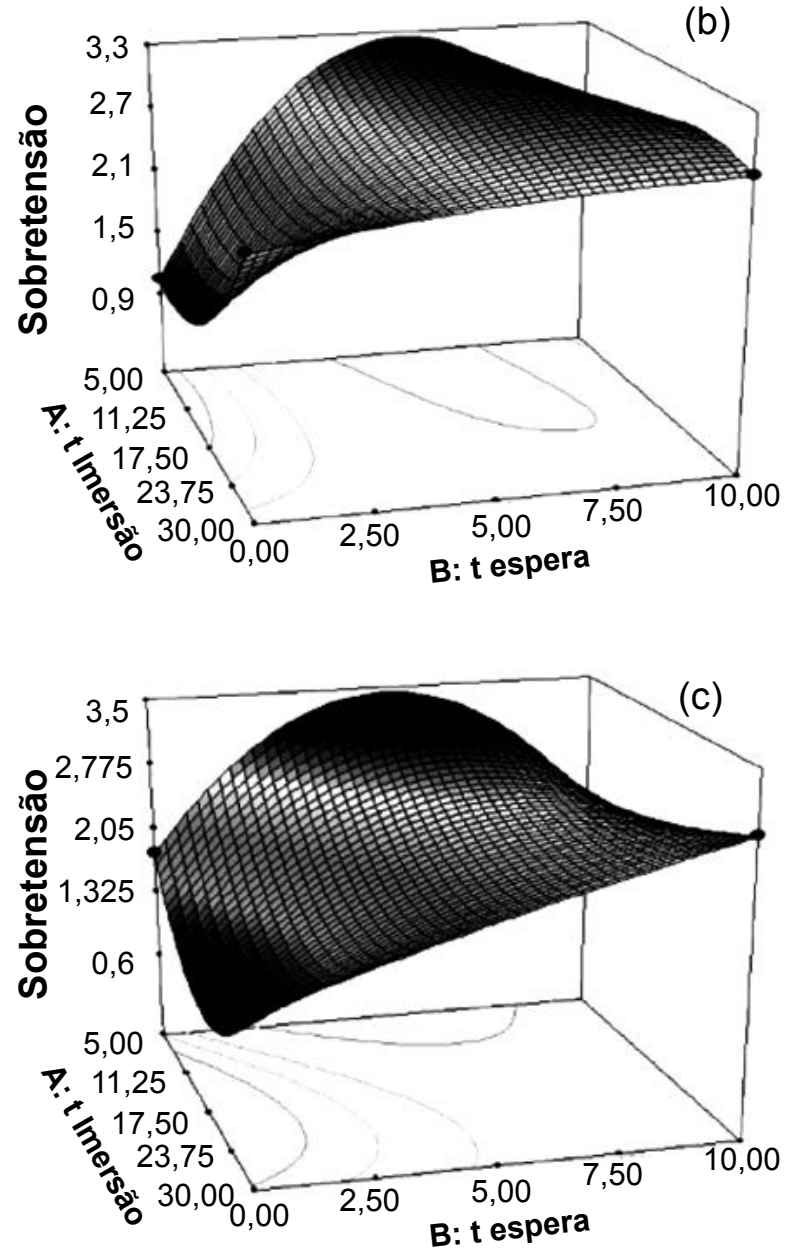

Figura 1: Superfície de resposta para a $\eta$ dos revestimentos de óxido de nióbio em função de imersão e tempo de espera, para as velocidades de (A) $6 \mathrm{~cm} / \mathrm{min}$, (B) $12 \mathrm{~cm} / \mathrm{min}$ e (C) $18 \mathrm{~cm} / \mathrm{min}$. [Figure 1: Surface response for $\eta$ of coated niobium oxide in immersion and timeout, for speed (A) $6 \mathrm{~cm} / \mathrm{min}$, (B) $12 \mathrm{~cm} / \mathrm{min}$ and (C) $18 \mathrm{~cm} / \mathrm{min}$.

formou um filme que contribuiu na proteção do metal.

A equação B representa as superfícies de respostas para os resultados experimentais do planejamento experimental $2^{3}$. 
$\eta=+2,53-0,44 * \mathrm{~A}+0,77 * \mathrm{~B}-0,21 * \mathrm{C}+0,078 * \mathrm{~A} * \mathrm{~B}-$

$0,19 * \mathrm{~A} * \mathrm{C}+0,077 * \mathrm{~A} * \mathrm{C}+0,26 * \mathrm{~A}^{2}$

$-0,79 * B^{2}+0,010 * C^{2}+0,20 * A * B * C-$

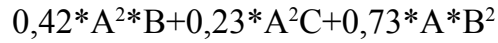

Para uma melhor compreensão das diferenças dos sistemas, foram realizadas análises de microscopia eletrônica

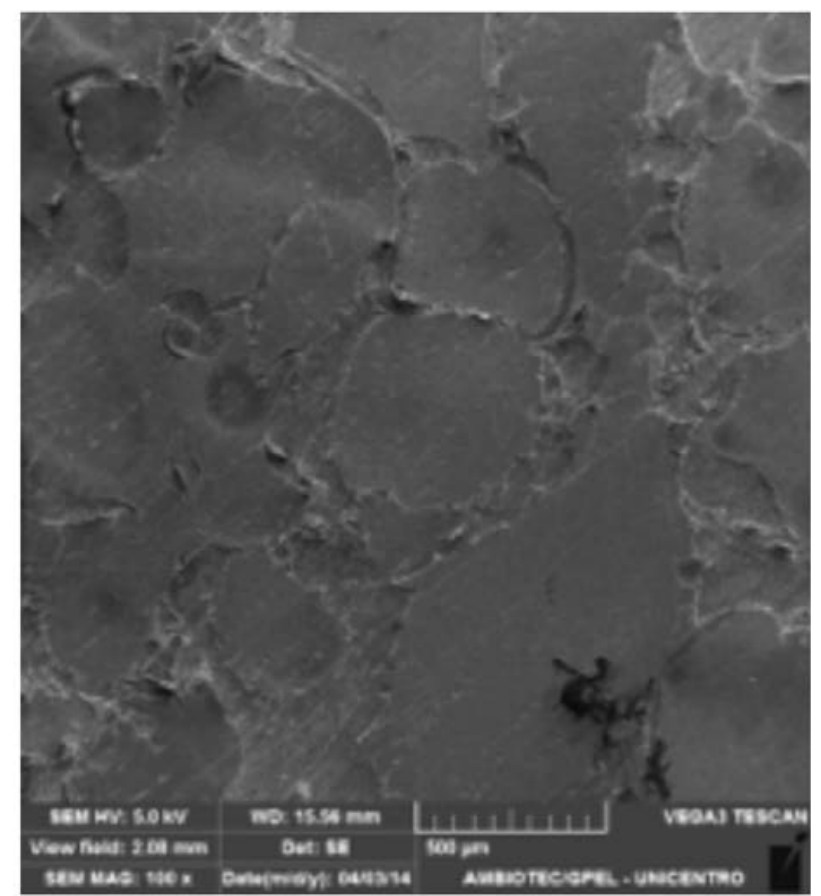

$2.1(\mathrm{~A})$

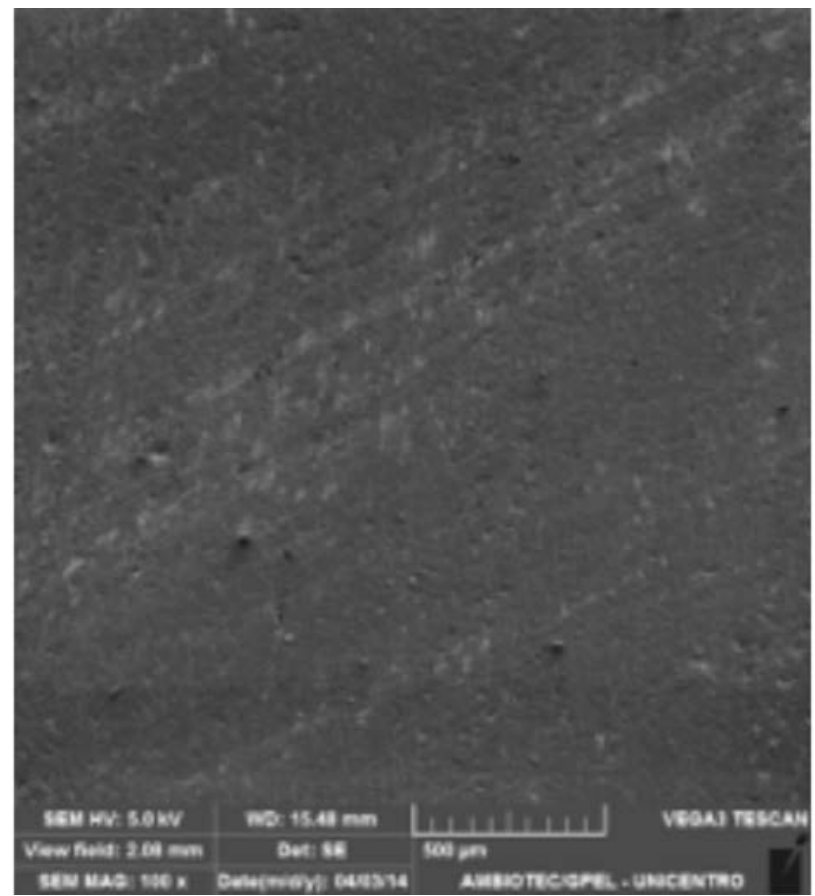

2.1 (B) de varredura das peças metálicas com a cerâmica depositada nas condições dos ensaios 14 e 8 , os quais apresentaram as respostas mais e menos satisfatórias, respectivamente. As imagens desses revestimentos estão apresentadas na Fig. 2.

Observa-se na Fig. 2-2.1(A) que a liga de alumínio 3003 revestida nas condições do ensaio 8 apresenta irregularidades em toda a superfície, ficando evidente na Fig. 2 -2.2 (A) que

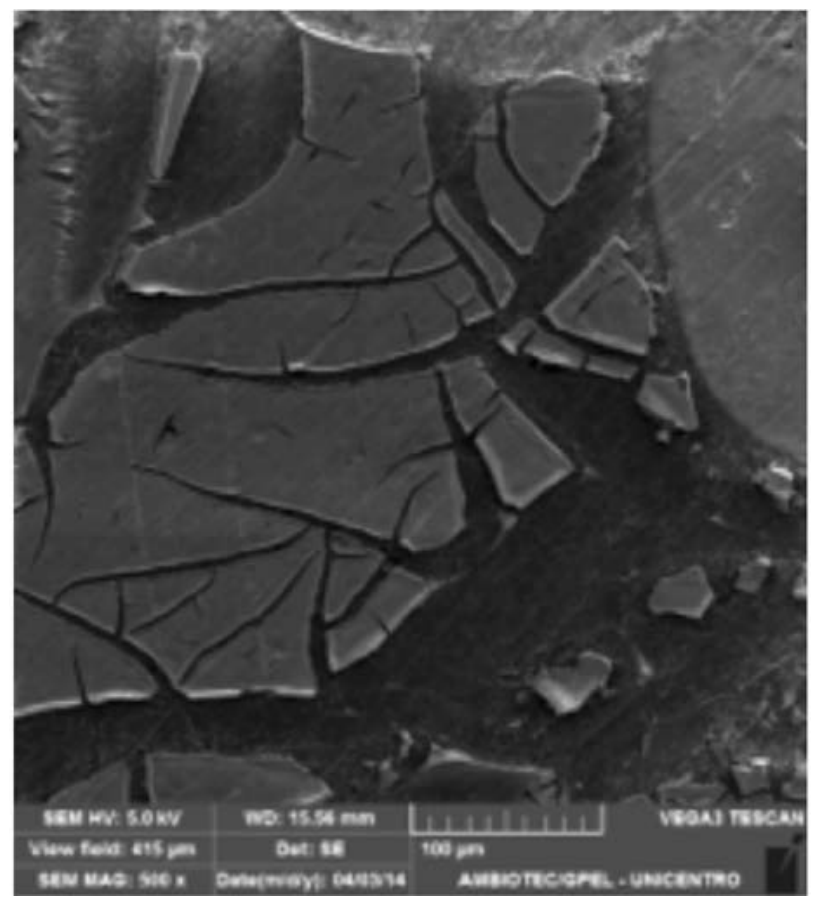

$$
2.2(\mathrm{~A})
$$

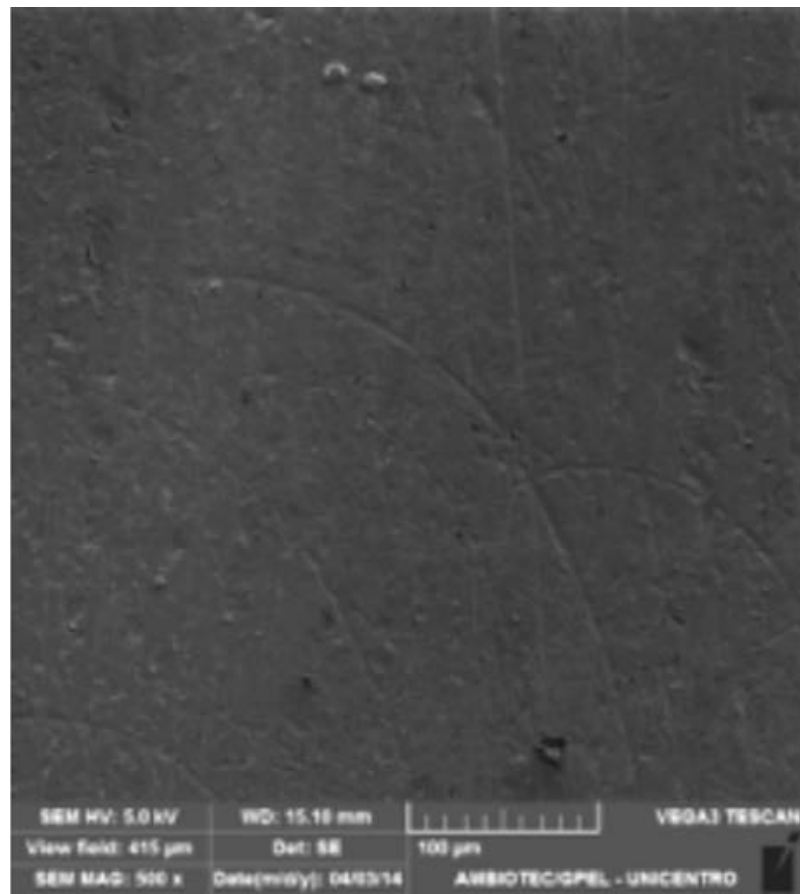

$2.2(\mathrm{~B})$

Figura 2: Micrografias obtidas por microscopia eletrônica de varredura da liga de alumínio 3003 com revestimento de óxido de nióbio depositado nas condições do ensaio 8 (A) e ensaio 14 (B), com aumentos de 100x (2.1) e 500x (2.2).

[Figure 2: Scanning electron microscopy micrographs of 3003 aluminum alloy with niobium oxide coating obtained in conditions in 8 (A) testing and 14 (B) testing, magnitude $100 x$ (2.1) and 500x (2.2).] 
$\mathrm{Nb} L \alpha 1$

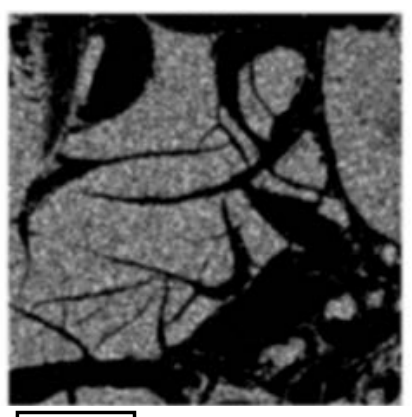

$\longdiv { 2 5 0 \mu \mathrm { m } }$

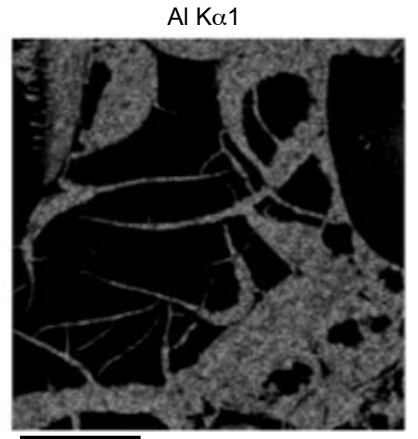

$\longdiv { 2 5 0 \mu \mathrm { m } }$
Figura 3: Mapeamento desenvolvido por EDS a partir da Fig. 2-2.2(A), 3(A) região clara indicando presença de $\mathrm{Nb}, 3$ (B) região clara indicando presença de Al.

[Figure 3: Mapping obtained via energy dispersive spectroscopy (EDS) from figure 2-2.2(A), (A) Clear region shows $\mathrm{Nb}$ presence, (B) Clear region shows Al presence.]
$\mathrm{Nb} L \alpha 1$

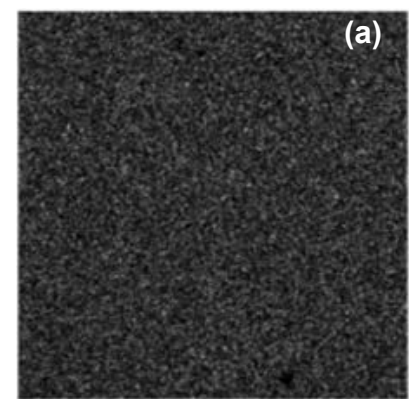

$\longdiv { 2 5 0 \mu \mathrm { m } }$

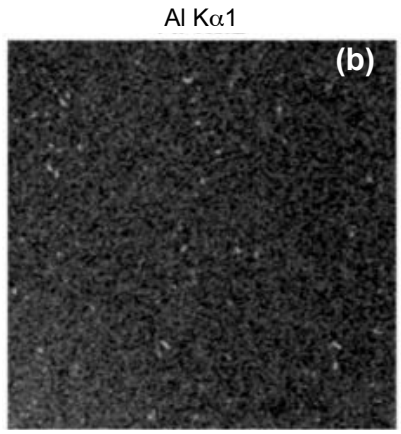

$\longdiv { 2 5 0 \mu \mathrm { m } }$
Figura 4: Mapeamento desenvolvido por EDS a partir da Fig. 2-2.2(B), 4(A) região clara indicando presença de $\mathrm{Nb}, 4$ (B) região clara indicando presença de Al.

[Figure 4: EDS mapping obtained from Fig. 2-2.2(B), 4(A) clear region shows $\mathrm{Nb}$ presence, $4(B)$ clear region shows Al presence.]

o revestimento possui grandes defeitos, que podem deixar o substrato metálico exposto ao meio corrosivo, o que é confirmado no mapeamento (Fig. 3(B)).

As regiões contendo $\mathrm{Al}$ e $\mathrm{Nb}$ são distintas, permanecendo uma grande área do substrato metálico desprotegida do filme cerâmico, tornando-se assim suscetível à corrosão. Essas deformações são geradas por dilatação térmica, uma vez que nas condições de depósito dessa cerâmica o revestimento era mais espesso, pois não houve eliminação do sol gel. Comportamento distinto é verificado para a liga de alumínio 3003 revestida nas condições do ensaio 14, mostrado nas Figs. 2-2.1B e 2.2B, onde toda a área apresenta um revestimento uniforme. Os elementos nióbio e alumínio estão dispostos de forma homogênea (Fig. 4), o que colabora para a cerâmica agir como uma barreira de proteção, impedindo o substrato metálico de entrar em contato com o meio agressivo.

Comparação eletroquímica do revestimento de óxido de nióbio depositado nas condições do ensaio 14, com o revestimento à base de $\mathrm{Cr}$ (III)

Para efeito de comparação com o revestimento utilizado industrialmente para proteção do substrato metálico, foram realizados ensaios com filme de $\mathrm{Cr}$ (III) que são apresentados nas Figs. 5 e 6.

Nota-se na Fig. 5(A) que um aumento nos valores de impedância foram medidos para as amostras revestidas com óxido de nióbio, quando comparado com o sistema revestido com Cr (III) e com substrato metálico, sugerindo uma maior resistência à corrosão para o sistema com revestimento de óxido de nióbio. Os diagramas de ângulo de fase de Bode da Fig. 5(B) indicam que para a liga de alumínio há uma constante de tempo em $10^{3} \mathrm{~Hz}$, e esta pode ser relacionada ao óxido formado na superfície do metal. Para o mesmo sistema, na região de $1 \mathrm{~Hz}$ há uma segunda constante de tempo que pode ser atribuída aos processos de transferência de carga na superfície metálica. Para a liga de alumínio revestida com $\mathrm{Cr}$ (III) observa-se duas constante de tempos:
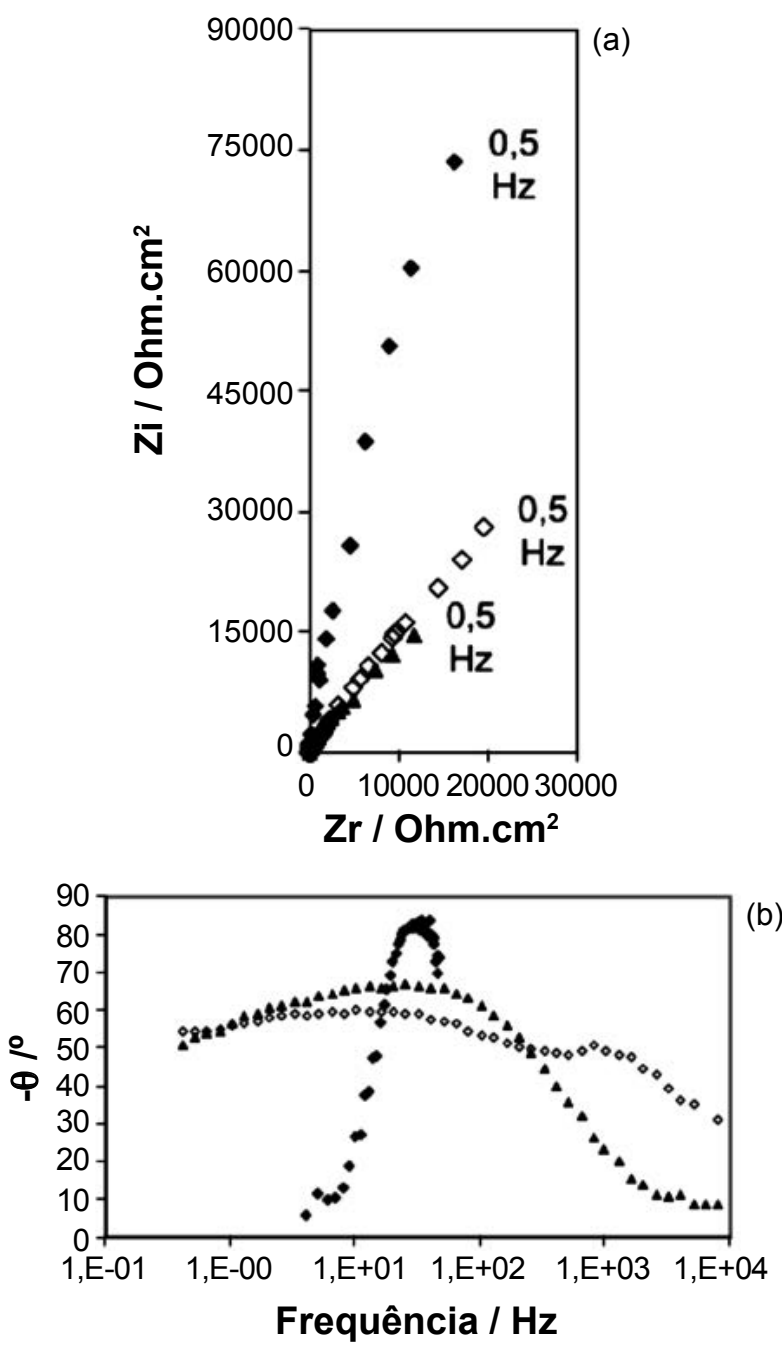

Figura 5: Diagramas de Nyquist (A) e de Bode (B) em $\mathrm{Na}_{2} \mathrm{SO}_{4} 0,5$ $\mathrm{mol} / \mathrm{L} \mathrm{em} \mathrm{pH} \mathrm{4,} \mathrm{com} 800 \mathrm{mg} / \mathrm{L}$ de $\mathrm{NaCl}$ para a liga da Alumínio 3003 sem revestimento $(\boldsymbol{\Delta})$, com revestido de óxido de nióbio $(\bullet)$ e Cr III $(\diamond)$.

[Figure 5: Nyquist (A) and Bode phase angle (B) diagrams obtained in $\mathrm{Na}_{2} \mathrm{SO}_{4} 0.5 \mathrm{~mol} / \mathrm{L}$ in $\mathrm{pH} 4$ with $800 \mathrm{mg} / \mathrm{L}$ in $\mathrm{NaCl}$ of 3003 aluminum alloy free coating $(\mathbf{\Delta})$, with niobium oxide coating (४) and $\mathrm{Cr}(\mathrm{III})(\diamond)$.] 
em $10^{3} \mathrm{~Hz}$, com ângulo de fase próximo de $50^{\circ}$, atribuída ao revestimento; e em $10 \mathrm{~Hz}$, associada aos processos de transferência de carga. No sistema com óxido de nióbio há uma constante de tempo entre $10 \mathrm{~Hz}$ e $10^{2} \mathrm{~Hz}$, em ângulo de fase próximo a $85^{\circ}$, referente à camada de óxido de nióbio do revestimento. A segunda constante de tempo aparece em

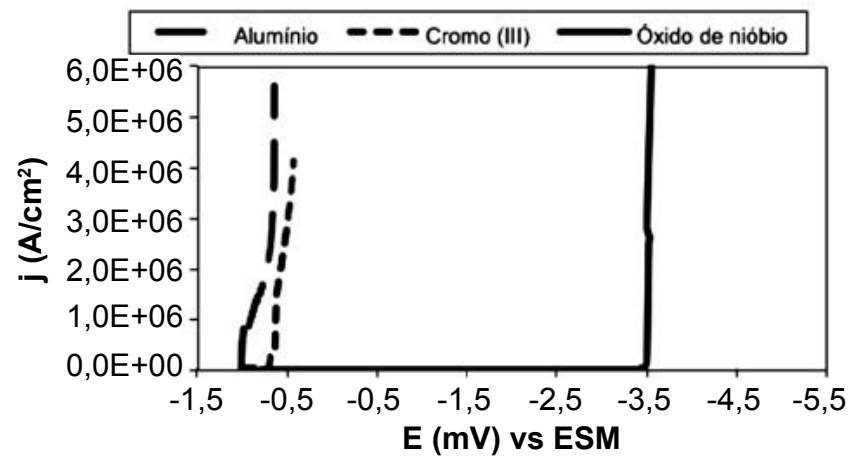

Figura 6: $\mathrm{PP}$ em $\mathrm{Na}_{2} \mathrm{SO}_{4} 0,5 \mathrm{~mol} / \mathrm{L} \mathrm{em} \mathrm{pH} \mathrm{4,} \mathrm{com} 800$ ppm de $\mathrm{NaCl}$ para a Liga da $\mathrm{Al} 3003$ sem e com revestido de nióbio e $\mathrm{Cr}$ (III).

[Figure 6: $\mathrm{PP}$ in $\mathrm{Na}_{2} \mathrm{SO}_{4} 0.5 \mathrm{~mol} / \mathrm{L}$ in $\mathrm{pH} 4$ with $800 \mathrm{mg} / \mathrm{L}$ in $\mathrm{NaCl}$ of 3003 aluminum alloy free coating, with niobium and $\mathrm{Cr}$ (III oxide coatings).]

$10 \mathrm{~Hz}$, associada aos processos de transferência de carga.

Os ensaios de PP para o substrato metálico sem e com revestimento de $\mathrm{Cr}$ (III) e óxido de nióbio estão apresentados na Fig. 6. O depósito do óxido de nióbio foi realizado nas condições do ensaio 14 do planejamento experimental $2^{3}$.

Nas curvas de PP (Fig. 6) é possível observar registro de duas regiões distintas presentes para o sistema revestido com óxido de nióbio e Cr (III). Para o revestimento de $\mathrm{Cr}$ (III) a primeira região aparece $\mathrm{em} n$ de $+280 \mathrm{mV}$, na qual um comportamento de baixa corrente é observado. A segunda região aparece em $\eta$ maiores que $+280 \mathrm{mV}$, na qual um aumento na densidade de corrente é verificado para o sistema com Cr (III). Comportamento semelhante é observado para o revestimento de óxido de nióbio, contudo o aumento da densidade de corrente foi retardado, em n próxima de $+4 \mathrm{~V}$ em relação ao potencial de corrosão, mostrando que o óxido de nióbio apresentou um efeito inibidor de corrosão para a liga de alumínio 3003 e superior ao revestimento de $\mathrm{Cr}$ (III).

\section{CONCLUSÕES}

O planejamento experimental se mostrou adequado para selecionar as melhores condições de depósito do sol gel à base de óxido de nióbio sobre liga de alumínio 3003 . As superfícies de respostas mostraram uma tendência no comportamento das $n$, sendo o tempo de emersão a variável que mais influenciou o sistema. O revestimento de óxido de nióbio depositado nas condições de velocidade $18 \mathrm{~cm} / \mathrm{min}$, 5 min de imersão e emersão, apresentou maiores valores de impedância e n, quando comparado com o revestimento de $\mathrm{Cr}$ (III) e com liga de $\mathrm{Al}$ 3003. A cerâmica à base de óxido de nióbio proposta neste trabalho apresenta uma alta resistência à corrosão, oferecendo uma maior eficiência inibidora do que revestimento à base de $\mathrm{Cr}$ (III).

\section{REFERÊNCIAS}

[1] G. J. T. Alves, A. L. Gallina, S. R. M. Antunes, S. M. Kurchaidt, P. R. P. Rodrigues, "Surface Treatments and Exposition", Anais Interfinish (2012) 32-40.

[2] M. Tussolini, C. Spagnol, G. J. T. Alves, M. Cunha, P. Rodrigues, Rev. Escola de Minas 63 (2010) 87-93.

[3] E. P. Banczek, M. Terada, P. R. P. Rodrigues, I. Costa, J. Mater. Eng. Perform. 22, 11 (2013) 3572-3583.

[4] M. Tussolini, P. Rodrigues, A. Viomar, A. Galina, M. Cunha, E. P. Banczek, Rev. Escola de Minas 66 (2013) 215 220.

[5] F. M. Queiroz, C. I. Elsner, C. R. Tomachuk, A. R. Di Sarli, I. Costa, Technol. Metal. Mater. Miner 10, 1 (2013) 8-15.

[6] G. S. Frankel, R. L. McCreery, Interface (2001) 34-38.

[7] J. W. Clark, R. L McCreery, J. Electrochem. Soc. 149 (2002) B379-B386.

[8] J. M. G. Lima, Ministério de Minas e Energia, Secretaria de geologia, mineração e transformação mineral (2010).

[9] T. Sreethawong, S. Ngamsinlapasathian, S. Yoshikawa, Mater. Lett. 78 (2012) 135-138.

[10] G. V. Tagliaferro, P. H. F. Pereira, L. Á. Rodrigues, M. L. C. P. Silva, Quim. Nova 34, 1 (2011) 101-105.

[11] A. L. C. Peixoto, G. L. J. P. da Silva, M. L. C. P. da Silva, Cerâmica 52, 324 (2006) 249-254.

[12] L. C. A. Oliveira, M. Gonçalves, D. Q. L. Oliveira, A. L. N. Guarieiro, Quim. Nova 30, 4 (2007) 925-929.

[13] O. Regis Junior, J. M. da. Silva, K. F. Portella, R. S. C. Pareces, Quim. Nova 25 (2009) 1517-1521.

[14] C. L. T. da Silva, J. L. Zotin, V. L. L. Camorim, A. C. Faro, M. L. M. Rocco, Quim. Nova 21 (1998) 157-163.

[15] A. A. S. Alfaya, L. T. Kubota, Quim. Nova 25, 5 (2002) 835-841.

[16] R. S. Hiratsuka, C. V. Santilli, S. H. Pulcinelli, Quim. Nova 18, 2 (1995) 171-180.

[17] E. J. Nassar, Y. Messaddeq, S. J. L. Ribeiro, Quim. Nova 25, 1 (2002) 27-31.

[18] S. R. Federman, V. T. L. Buono, W. L. Vasconcelos, Cerâmica 55, 334 (2009) 177-180.

[19] G. L. Lucena, J. J. N. Souza, A. S. Maia, L. E. B. Soledade, E. Longo, A. G. Souza, I. M. G. Santos, Cerâmica 59, 350 (2013) 249-253.

[20] K. Zheng, Y. Liu, Z. Liu, Z. Chen, W. Qin, Dalton Transactions 42 (2013) 5159-5166.

[21] T. Grzyb, S. Lis, Inorganic Chem. 50 (2011) 8112-8120. [22] J. H. G. Rangel, F. M. L. Pontes, E. R. Leite, J. A. Varela, Cerâmica 46, 300 (2000) 181-185.

[23] R. G. Freitas, R. T. S. Oliveira, M. C. Santos, L. O. S. Bulhões, E. C. Pereira, Mater. Let. 60 (2006) 1906-1910.

[24] M. Galceran, M. C. Pujol, M. Aguiló, F. Dáaz, J. SolGel Sci. Technol. 42 (2007) 79-88.

[25] I. D. Santos, J. C. Afonso, A. J. B. Dutra, Rev. Mater. 14, 3 (2009) 1015-1027.

[26] N. Cioatera, A. Samide, A. Maxut, R. N. Vannier, M. 
Traisnel, Rev. Roum. Chim. 56 (2011) 1003-1009.

[27] J. R. M Hoyos, F. L. Zabotto, D. Garcia, R. H. G. A. Kiminami, Cerâmica 59, 351 (2013) 360-365.

[28] S. B. Galvão, A. C. Lima, S. N. de Medeiros, J. M. Soares, C. A. Paskocimas, Mater. Lett. 115 (2013) 38-41.
[29] L. F. A. Campos, R. R. Menezes, D. Lisboa, L. N. L. Santana, G. A. Neves, H. C. Ferreira, Cerâmica 53, 328 (2007) 373-380.

[30] C. H. M. Rodrigues, M. Toniato, F. G. Souza Jr, H. R. Paes Jr, Cerâmica 59, 351 (2013) 451-459.

(Rec. 02/05/2014, Ac. 08/05/2014) 